\title{
Comparación histoquímica de albura y duramen de tres especies de Quercus
}

\author{
Laura Rivera Nava ${ }^{1}$ \\ Alejandra Quintanar Isaías ${ }^{1}$ \\ Carmen de la Paz Pérez Olvera ${ }^{1}$
}

\begin{abstract}
RESUMEN
Se describen las características histoquímicas cualitativas y morfológicas cuantitativas de algunas células que componen la albura y el duramen de Quercus glabrescens, $Q$. potosina y $Q$. eduardii que fueron colectados en el municipio de Huayacocotla, Veracruz. Los resultados indican que las alburas son muy semejantes en sus características histoquímicas y en las tendencias cuantitativas, pero las de duramen presentan diferencias en: tipo de fibras gelatinosas, depósitos violáceos de polisacáridos en cavidades de fibras y parénquima radial y en este tipo celular, la presencia de algunos procesos pétreos en la madera de $Q$. potosina. Se discute el concepto de calidad de pared, la relación entre el tipo de componentes químicos y dimensiones celulares.
\end{abstract}

PALABRAS CLAVE:

Madera, histoquímica, pared celular, Quercus, México.

\section{INTRODUCCION}

Los componentes químicos de las paredes celulares que conforman el xilema secundario varían dependiendo de la especie, así como del proceso de muerte celular, el que puede culminar tanto en la transformación de los componentes

\begin{abstract}
Histochemical and some quantitative features of sapwood and heartwood cells of Quercus glabrescens, $Q$. potosina y $Q$. eduardii are described. The trees were collected in different sites of Huayacocotla, in Veracruz State. The results show that sapwood of the species are very similar in histochemical and quantitative trends but heartwood differ on chemical deposits of gelatinous fibers, and violet deposits of polisacharids present on rays and also the petrification processes in $Q$. potosina rays. Cell wall quality and the relationship between dimensions and types of chemical compounds are discussed as well.
\end{abstract}

KEY WORDS:

Wood, histochemistry, cell wall, Quercus, México.

químicos de las paredes como en la producción de extractivos, como gomas, resinas, taninos o inclusiones minerales, que se depositan en las paredes o en las cavidades celulares. Estos componentes pueden ser reconocidos in situ, mediante la utilización de métodos histoquímicos específicos. Estos tienen como fundamento 
las reacciones en color de los distintos componentes químicos de la pared celular, de manera que, las intensidades de las reacciones para distinguir celulosa, lignina, pectinas, polisacáridos y diferentes tipos de extractivos como taninos, catequinas, leucoantocianidinas, resinas, gomas 0 ácidos grasos dependen de las proporciones de dichos componentes (Quintanar et al., 1997).

Todos estos tipos de componentes químicos presentes en elementos celulares verticales y horizontales influyen en las propiedades físicas y mecánicas de las paredes celulares, esto es en la rigidez, capacidad de deformación, capacidad de contraerse longitudinal o transversalmente, etc. (Vincent, 1982). Aunque las fibras gelatinosas pueden influir en estas propiedades, no existe literatura que oriente sobre las propiedades que modifican estas células al conjunto del tejido. Existe poca literatura referente al análisis de los componentes químicos de las paredes celulares de la madera utilizando métodos histoquímicos (Baqui et al., 1979; Shah et al., 1981; Datta y Kumar, 1987; Krishnamurthy, 1988; Donaldson, 1992; Quintanar et al., 1997).

La madera de Quercus es muy apreciada y valorada en otros países y le han dado usos muy diversos. Por sus características de veteado y textura ha sido muy usada en la manufactura de gabinetes finos, muebles, chapa y partes de instrumentos musicales, además de tener buena respuesta al acabado cuando se trabaja. Se ha utilizado en partes de barcos $y$ embarcaciones, como las quillas $y$ armaduras, así como en pisos de vehículos de motor y tarimas de carga y descarga, usos que requieren de buena dureza y resistencia. Se le ha considerado como la principal madera para pisos y tonelería, dadas sus características de resistencia al desgaste, presencia de tílides y alta resistencia a la pudrición (De la Paz Pérez, 1982). Sin embargo en nuestro país, a pesar de ser un recurso forestal abundante y con gran potencial tecnológico lo han destinado para leña y carbón básicamente. Las opciones en el aprovechamiento de sus especies son escasas, debido en parte al desconocimiento de las propiedades tecnológicas que poseen las mismas. Las pruebas histoquímicas cualitativas, junto con algunas dimensiones de los elementos celulares, proporcionan información útil sobre la calidad de la madera a nivel microscópico.

\section{OBJETIVO}

Este trabajo tiene como objetivo contribuir al conocimiento de las características histoquímicas de Quercus glabrescens Benth. y $Q$. potosina Trel., pertenecientes al subgénero Leucobalanus (encinos blancos) y de Quercus eduardii Trel., perteneciente al subgénero Erythrobalanus (encinos rojos), así como de algunos aspectos morfológicos cuantitativos para discutir la relación entre los tipos de componentes químicos, su repercusión en la calidad de la madera y algunas posibles estrategias de defensa, competencia o supervivencia desarrolladas por estas especies ante el ambiente en el que habitan.

\section{MATERIALES Y METODOS}

Los árboles estudiados en este trabajo provienen de dos localidades del municipio de Huayacocotla, Veracruz. Q. glabrescens y $Q$. eduardii fueron colectados en un bosque mesófilo de montaña y $Q$. potosina se colectó en bosque adyacente al anterior en el que la desertificación es un problema serio. Los ejemplares de herbario y las muestras de xiloteca (tablillas) quedaron registrados en el Herbario Metropolitano UAMIZ (Tabla 1).

De cada individuo se cortaron dos rodajas de $2 \mathrm{~cm}$ de grosor a $1.40 \mathrm{~m}$ de altura, de estas se obtuvieron cubos de $2 \mathrm{x}$ $2 \mathrm{~cm}$ de albura y duramen. Los cubos 
fueron ablandados en una mezcla de agua destilada, glicerol y polietilenglicol durante dos horas. De cada muestra se hicieron cortes transversales, tangenciales y radiales con grosores de 16 a $22: \mathrm{m}$. Por cada prueba histoquímica se realizaron diez repeticiones como mínimo en cada uno de los cortes y para cada tipo celular descrito. Los almidones y los lípidos fueron teñidos con el método de Johansen (1940). Los otros métodos histoquímicos utilizados en este estudio están descritos en detalle en Krishnamurthy (1988). Para la detección de almidones y lípidos se usaron lugol y Sudán III. Para teñir diferencialmente celulosa (azul), hemicelulosas (morado, azul lila, rosa-violeta) y lignina (azul turquesa), se usó azul de toluidina-O. Para diferenciar celulosa (azul a violeta) de lignina (amarillo a naranja) se siguió el método de zinc-cloroiodo, aunque pueden presentarse carbohidratos de color violáceo en el lumen de las células. Para observar solo pectatos de calcio (negro a azul oscuro) se utilizó el método de ácido tánico-cloruro férrico. La presencia de únicamente pectinas esterificadas se probó por el método de clorhidrato de hidroxilamina alcalina (rosarojo). Se usó fluroglucinol para teñir específicamente lignina (rojo a violeta). Los componentes fenólicos fueron teñidos específicamente por el método de vainillina; las catequinas y leucoantocianidinas son vistas como depósitos rojos, mientras que sus oligómeros se observan como depósitos pardo-rojizos; las paredes celulares se observan de color pardo si presentan polifenoles.

Se tomaron fotografías de las diferentes reacciones de los tejidos y el incremento en la intensidad de las reacciones se ponderó cualitativamente usando el símbolo (+).

En este punto se designó como:

$+\quad=$ presencia escasa;

$++\quad=$ presencia mediana; $y$

$+++=$ presencia intensa
Las tonalidades se han representado en la literatura como intensidades y así se toman en este trabajo. De ahí que al identificar una intensidad como escasa, mediana o intensa (tablas 3 a 5), se está indicando la presencia de los componentes químicos en esa misma magnitud.

También se elaboraron preparaciones fijas para realizar las siguientes cuantificaciones: longitud y diámetro de fibras, vasos, traqueidas y fibrotraqueidas; altura y anchura de las células de los paréquimas axial y radial, así como el grosor de las paredes de todos estos tipos celulares. Adicionalmente, se hizo una revisión de la colección de preparaciones de las especies de Quercus que se tienen en el Laboratorio de Anatomía y Tecnología de la Madera de la UAM-I para verificar la presencia de fibras gelatinosas en el género Quercus (Tabla 2).

\section{RESULTADOS}

1. Quercus glabrescens Benth. (Leucobalanus) (Tablas 3, 6a, 6b y 6c; Lámina 1).

El duramen de esta especie se caracterizó por presentar tres zonas de forma irregular, pero perfectamente diferenciadas. La Zona I de color grisrosado (Z-I), la Zona II de color castaño oscuro (Z-II) y la Zona III de color castaño claro (Z-III) (Figura 1). El análisis de la madera se hizo considerando estas zonas como distintas entre sí.

2. Quercus potosina Trel. (Leucobalanus) (Tablas 4, 6a, 6b y 6c; Lámina 2).

3. Quercus eduardii Trel. (Erythrobalanus) (Tablas 5, 6a, 6b y 6c; Lámina 3). 
Tabla 1. Algunos datos de los encinos estudiados

\begin{tabular}{||l|l|l|c|c||}
\hline \hline \multirow{2}{*}{ NOMBRE CIENTIFICO } & \multicolumn{1}{|c|}{ SUBGENERO } & NOMBRE & \multicolumn{2}{c||}{ REGISTRO } \\
& \multicolumn{1}{|c||}{ COMUN } & Xiloteca & Herbario \\
\hline Quercus glabrescens & Leucobalanus (encino blanco) & Quebracho & M-96 & 41824 \\
\hline Quercus potosina & Leucobalanus (encino blanco) & Quebracho & M-100 & 41828 \\
\hline Quercus eduardii & Erythrobalanus (encino rojo) & Manzanillo & M-101 & 41829 \\
\hline
\end{tabular}

Tabla 2. Presencia de fibras gelatinosas en Quercus spp.

\begin{tabular}{|c|c|c|c|c|c|}
\hline ESPECIE & $\begin{array}{l}\text { REGISTRO } \\
\text { XILOTECA } \\
\end{array}$ & \begin{tabular}{|c|c|c|} 
ESTADO DE \\
COLECTA
\end{tabular} & ESPECIE & $\begin{array}{l}\text { REGISTRO } \\
\text { XILOTECA } \\
\end{array}$ & $\begin{array}{l}\text { ESTADO DE } \\
\text { COLECTA } \\
\end{array}$ \\
\hline \multirow{2}{*}{ 1. Q. candicans } & $\mathrm{X}-406$ & Michoacán & 10. Q. convallata & $\mathrm{X}-318$ & Durango \\
\hline & $X-506$ & Michoacán & 11. Q. excelsa & $\mathrm{X}-454$ & Jalisco \\
\hline \multirow{3}{*}{ 2. Q. castanea } & M-50 & Jalisco & \multirow{4}{*}{ 12. Q. glabrescens } & M-67 & \multirow{2}{*}{ Puebla } \\
\hline & $X-507$ & Michoacán & & M-93 & \\
\hline & $M-74$ & Puebla & & M-96 & \multirow{2}{*}{ Veracruz } \\
\hline 3. Q. crassifolia & $\mathrm{M}-70$ & Jalisco & & $\mathrm{M}-98$ & \\
\hline 4. Q. durifolia & $x-376$ & Durango & 13. Q. laeta & $X-375$ & Durango \\
\hline \multirow{2}{*}{ 5. Q. eduardii } & M-101 & \multirow{2}{*}{ Veracruz } & \multirow{2}{*}{ 14. Q. obtusata } & $\mathrm{X}-403$ & \multirow{2}{*}{ Michoacán } \\
\hline & M-101 & & & $\mathrm{X}-505$ & \\
\hline \multirow{2}{*}{ 6. Q. fulva } & $\mathrm{X}-373$ & \multirow{2}{*}{ Durango } & \multirow{2}{*}{ 15. Q. potosina } & $\mathrm{X}-377$ & \multirow{2}{*}{ Durango } \\
\hline & $\mathrm{X}-373$ & & & $\mathrm{X}-377$ & \\
\hline 7. Q. ghiesbreghtii & M-99 & Veracruz & 16. Q. resinosa & $\begin{array}{l}\mathrm{X}-508 \\
\end{array}$ & Michoacán \\
\hline 8. Q. skinnerii & $\mathrm{X}-540$ & Chiapas & 17. Q. rugosa & $\mathrm{X}-444$ & Chiapas \\
\hline \multirow{2}{*}{ 9. Q. uxoris } & \multirow{2}{*}{$X-450$} & \multirow{2}{*}{ Jalisco } & \multirow{2}{*}{ 18. Q. xalapensis } & M-97A & \multirow{2}{*}{ Veracruz } \\
\hline & & & & M-97D & \\
\hline
\end{tabular}

La nomenclatura para los encabezados de las tablas 3 a 5 es la siguiente:
AT1 = azul de toluidina (lignina)
AT2 = azul de toluidina (celulosa)
hem $=$ presencia de hemicelulosas
$\mathrm{ZCl}=$ zinc-cloro-iodo (celulosa)
$\mathrm{cV}=$ carbohidratos violáceos
$\mathrm{AcT}-\mathrm{CF}=$ ácido tánico - cloruro férrico

(pectatos de calcio)

$\mathrm{CHA}=$ clorhidrato de hidroxilamina alcalina (pectinas esterificadas)

$\mathrm{F}=$ fluoroglucinol (lignina)

V1 = vainillina (compuestos fenólicos pardos)

V2 = vainillina (depósitos pardo rojizos)

$>_{+}=$aumenta la intensidad de la reacción 
Tabla 3. Análisis histoquímico de Quercus glabrescens Benth. (Leucobalanus).

\begin{tabular}{|c|c|c|c|c|c|c|c|c|c|c|}
\hline Reactivo & AT1 & AT2 & $\mathrm{ZCl}$ & AcT-CF & $\mathrm{CHA}$ & $\mathrm{F}$ & V1 & V2 & Lugol & Sudán II \\
\hline \multicolumn{11}{|c|}{ Células de albura } \\
\hline Fibras & ++ & + & - & + & + & + & + & - & - & - \\
\hline Fibras gelatinosas & ++ & hem & - & +++ & + & + & + & - & - & - \\
\hline Traqueidas vasicéntricas & ++ & - & + & + & + & +++ & + & - & - & - \\
\hline Vasos & ++ & - & - & + & + & +++ & + & - & - & - \\
\hline Parénquima axial & + & ++ & $\mathrm{cV}$ & + & + & + & + & - & +++ & - \\
\hline Radios multiseriados & ++ & - & $\mathrm{cV}$ & + & + & ++ & + & - & +++ & + \\
\hline Radios uniseriados & - & +++ & $\mathrm{cV}$ & + & + & + & + & - & +++ & + \\
\hline Lámina media & - & hem & - & +++ & - & + & - & - & - & - \\
\hline \multicolumn{11}{|c|}{ Células de duramen } \\
\hline \multicolumn{11}{|c|}{ Z-I } \\
\hline Fibras & ++ & + & - & + & + & + & + & - & - & - \\
\hline Fibras gelatinosas & + & hem & + & +++ & + & + & + & - & - & - \\
\hline Traqueidas vasicéntricas & ++ & ++ & + & + & + & ++ & + & - & - & - \\
\hline Vasos & + & +++ & + & + & + & +++ & + & - & - & - \\
\hline Tílides & + & ++ & + & ++ & + & + & + & - & - & - \\
\hline Parénquima axial & + & ++ & $\mathrm{cV}$ & ++ & + & + & + & - & - & - \\
\hline Radios multiseriados & ++ & + & $\mathrm{cV}$ & ++ & + & + & + & - & - & - \\
\hline Radios uniseriados & + & ++ & $\mathrm{CV}$ & ++ & + & + & + & - & - & - \\
\hline Lámina media & - & hem & - & +++ & - & + & - & - & - & - \\
\hline \multicolumn{11}{|c|}{ Z-II } \\
\hline Fibras & +++ & - & - & + & + & +++ & - & - & - & - \\
\hline Fibras gelatinosas & +++ & - & - & + & + & +++ & - & - & - & - \\
\hline Traqueidas vasicéntricas & +++ & - & - & + & + & +++ & + & - & - & - \\
\hline Vasos & +++ & + & - & + & + & +++ & + & - & - & - \\
\hline Tílides & ++ & + & - & ++ & + & ++ & + & - & - & - \\
\hline Parénquima axial & ++ & + & - & ++ & + & ++ & + & - & - & - \\
\hline Radios multiseriados & +++ & - & - & + & + & ++ & + & - & - & - \\
\hline Radios uniseriados & - & +++ & - & + & + & ++ & + & - & - & - \\
\hline Lámina media & - & hem & - & +++ & - & ++ & - & - & - & - \\
\hline \multicolumn{11}{|c|}{ Z-III } \\
\hline Fibras & ++ & ++ & - & + & + & + & + & - & - & - \\
\hline Fibras gelatinosas & + & hem & + & ++ & + & + & + & - & - & - \\
\hline Traqueidas vasicéntricas & ++ & + & + & + & + & ++ & + & - & - & - \\
\hline Vasos & ++ & + & + & + & + & ++ & + & - & - & - \\
\hline Tílides & + & ++ & + & ++ & + & + & + & - & - & - \\
\hline Parénquima axial & + & ++ & + & ++ & + & + & + & - & - & - \\
\hline Radios multiseriados & + & ++ & ++ & ++ & + & ++ & + & - & - & - \\
\hline Radios uniseriados & + & +++ & - & ++ & + & + & + & - & - & - \\
\hline Lámina media & - & hem & - & +++ & - & + & - & - & - & - \\
\hline
\end{tabular}




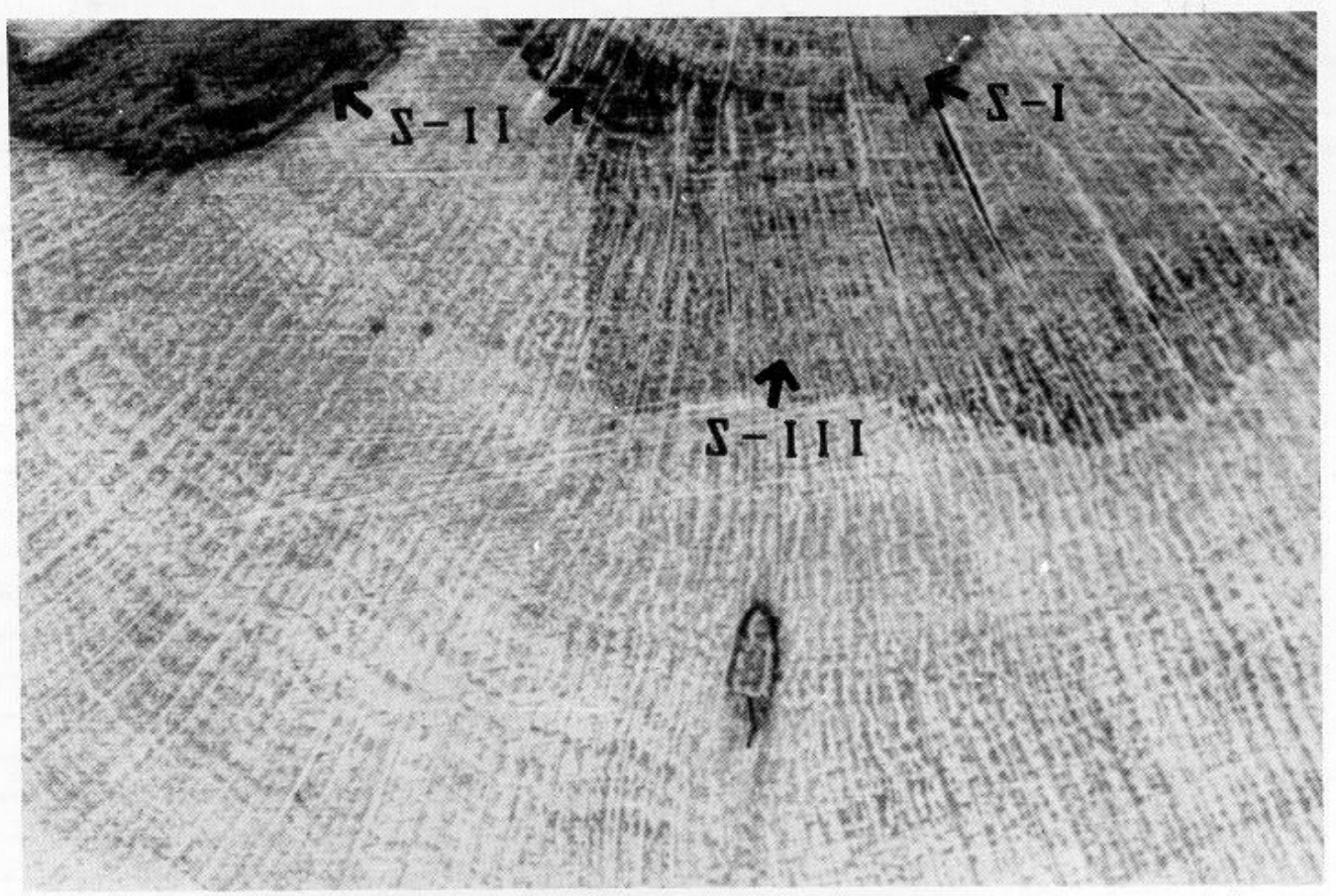

Figura 1. Tres zonas diferentes del duramen de Quercus glabrescens Benth.

Tabla 4. Análisis histoquímico de Quercus potosina Trel. (Leucobalanus).

\begin{tabular}{|c|c|c|c|c|c|c|c|c|c|c|}
\hline Reactivo & 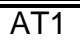 & AT2 & $\overline{\mathrm{ZCl}}$ & $\overline{\mathrm{AcT}-\mathrm{CF}}$ & $\mathrm{CHA}$ & $\overline{\mathrm{F}}$ & $\overline{\mathrm{V} 1}$ & $\overline{\mathrm{V} 2}$ & Lugol & Sudán III \\
\hline \multicolumn{11}{|c|}{ Células de albura } \\
\hline Fibras & ++ & + & $\mathrm{cV}$ & + & + & ++ & + & - & - & - \\
\hline Traqueidas vasicéntricas & + & + & $\mathrm{cV}$ & ++ & + & +++ & + & - & - & - \\
\hline Vasos & ++ & + & - & + & + & +++ & + & - & - & - \\
\hline Tílides & + & ++ & cV & + & + & ++ & + & - & ++ & + \\
\hline Parénquima axial & + & ++ & $\mathrm{CV}$ & + & + & - & + & - & ++ & + \\
\hline Radios multiseriados & + & - & $\mathrm{cV}$ & + & + & + & + & ++ & ++ & + \\
\hline Radios uniseriados & - & ++ & $\mathrm{CV}$ & + & + & - & + & ++ & ++ & + \\
\hline Lámina media & - & - & - & +++ & - & - & - & - & - & - \\
\hline \multicolumn{11}{|c|}{ Células de duramen } \\
\hline Fibras & +++ & + & + & + & + & + & + & - & - & - \\
\hline Traqueidas vasicéntricas & +++ & Hem & + & + & + & ++ & + & - & - & - \\
\hline Vasos & ++ & + & - & + & + & ++ & + & - & - & - \\
\hline Tílides & ++ & Hem & + & + & + & + & + & + & - & - \\
\hline Parénquima axial & + & +++ & + & + & + & + & + & - & - & - \\
\hline Radios multiseriados & +++ & - & + & + & + & - & + & + & - & - \\
\hline Radios uniseriados & - & +++ & + & + & + & - & + & + & - & - \\
\hline Lámina media & - & - & - & +++ & - & - & - & - & - & - \\
\hline
\end{tabular}


Tabla 5. Análisis histoquímico de Quercus eduardii Trel. (Erythrobalanus).

\begin{tabular}{|c|c|c|c|c|c|c|c|c|c|c|}
\hline Reactivo & AT1 & AT2 & $\overline{\mathrm{ZCI}}$ & AcT-CF & $\overline{\mathrm{CHA}}$ & $\overline{\mathrm{F}}$ & $\overline{\mathrm{V} 1}$ & V2 & Lugol & Sudán III \\
\hline \multicolumn{11}{|c|}{ Células de albura } \\
\hline Fibras & ++ & - & + & + & + & + & + & - & - & - \\
\hline Fibras gelatinosas & ++ & hem & + & ++ & + & + & + & - & - & - \\
\hline Traqueidas vasicéntricas & ++ & + & - & + & + & ++ & + & - & - & - \\
\hline Vasos & ++ & + & - & - & + & +++ & + & - & - & - \\
\hline Tílides & + & ++ & - & + & + & ++ & + & + & - & - \\
\hline Parénquima axial & + & ++ & $\mathrm{cV}$ & + & + & - & + & + & +++ & + \\
\hline Radios multiseriados & ++ & - & $\mathrm{cV}$ & + & + & ++ & + & + & +++ & + \\
\hline Radios uniseriados & - & ++ & - & + & + & - & + & + & - & + \\
\hline Lámina media & - & - & - & ++ & - & - & - & - & - & - \\
\hline Im fibras & - & hem & - & ++ & - & + & - & - & - & - \\
\hline \multicolumn{11}{|c|}{ Células de duramen } \\
\hline Fibras & +++ & hem & + & + & + & ++ & + & + & - & - \\
\hline Fibras gelatinosas & ++ & hem & - & + & + & ++ & + & - & - & - \\
\hline Traqueidas vasicéntricas & +++ & + & - & + & + & ++ & + & - & - & - \\
\hline Vasos & +++ & ++ & - & - & + & +++ & + & + & - & - \\
\hline Tílides & + & +++ & + & + & + & ++ & + & + & - & - \\
\hline Parénquima axial & ++ & + & + & + & + & + & + & + & - & - \\
\hline Radios multiseriados & ++ & ++ & + & + & + & +++ & + & +++ & - & - \\
\hline Radios uniseriados & - & ++ & + & + & + & ++ & + & ++ & - & - \\
\hline Lámina media & - & - & - & +++ & - & - & - & - & - & - \\
\hline ఏm fibras & - & hem & - & +++ & - & + & - & - & - & - \\
\hline
\end{tabular}

Tabla 6a. Aspectos cuantitativos de fibras y vasos de Quercus glabrescens, Quercus potosina y Quercus eduardii.

\begin{tabular}{|c|c|c|c|c|c|c|}
\hline \multirow[b]{2}{*}{ ESPECIE } & \multicolumn{3}{|c|}{$\overline{\text { FIBRA }}$} & \multicolumn{3}{|c|}{ VASOS } \\
\hline & $\begin{array}{c}\text { LONGITUD } \\
: \mathrm{m}\end{array}$ & $\begin{array}{c}\text { DIAMETRO } \\
: \mathrm{m}\end{array}$ & $\begin{array}{c}\text { GROSOR } \\
\text { DE PARED } \\
: \mathrm{m}\end{array}$ & $\begin{array}{c}\text { LONGITUD } \\
: \mathrm{m}\end{array}$ & $\begin{array}{c}\text { DIAMETRO } \\
: m\end{array}$ & $\begin{array}{c}\text { GROSOR } \\
\text { DE PARED } \\
: m\end{array}$ \\
\hline \multirow{2}{*}{ Quercus glabrescens } & $\begin{array}{c}1445 \\
(1112-1728)\end{array}$ & $\begin{array}{c}21 \\
(18-30)\end{array}$ & $\begin{array}{c}7 \\
(6-12)\end{array}$ & $\begin{array}{c}520 \\
(344-744)\end{array}$ & $\begin{array}{c}162 \\
(50-280)\end{array}$ & $\begin{array}{c}7 \\
(6-10)\end{array}$ \\
\hline & $\begin{array}{c}1391 \\
(1064-1688)\end{array}$ & $\begin{array}{c}21 \\
(16-24)\end{array}$ & $\begin{array}{c}8 \\
(6-11)\end{array}$ & $\begin{array}{c}490 \\
(316-680)\end{array}$ & $\begin{array}{c}150 \\
(48-230)\end{array}$ & $\begin{array}{c}9 \\
(8-10)\end{array}$ \\
\hline \multirow{2}{*}{ Quercus potosina } & $\begin{array}{c}705 \\
(464-1048)\end{array}$ & $\begin{array}{c}19 \\
(16-24)\end{array}$ & $\begin{array}{c}8 \\
(6-10)\end{array}$ & $\begin{array}{c}331 \\
(160-560)\end{array}$ & $\begin{array}{c}127 \\
(44-222\end{array}$ & $\begin{array}{c}8 \\
(6-10)\end{array}$ \\
\hline & $\begin{array}{c}928 \\
(544-1224)\end{array}$ & $\begin{array}{c}19 \\
(16-26)\end{array}$ & $\begin{array}{c}7 \\
(6-10)\end{array}$ & $\begin{array}{c}359 \\
(216-592)\end{array}$ & $\begin{array}{c}126 \\
(50-210)\end{array}$ & $\begin{array}{c}7 \\
(4-10)\end{array}$ \\
\hline \multirow{2}{*}{ Quercus eduardii } & $\begin{array}{c}1372 \\
(800-1808)\end{array}$ & $\begin{array}{c}23 \\
(18-34)\end{array}$ & $\begin{array}{c}7 \\
(5-10)\end{array}$ & $\begin{array}{c}608 \\
(392-856)\end{array}$ & $\begin{array}{c}153 \\
(100-370)\end{array}$ & $\begin{array}{c}10 \\
(8-12)\end{array}$ \\
\hline & $\begin{array}{c}1323 \\
(1048-1744)\end{array}$ & $\begin{array}{c}22 \\
(16-28)\end{array}$ & $\begin{array}{c}6 \\
(4-8)\end{array}$ & $\begin{array}{c}586 \\
(360-800)\end{array}$ & $\begin{array}{c}173 \\
(56-326)\end{array}$ & $\begin{array}{c}7 \\
(6-10)\end{array}$ \\
\hline
\end{tabular}

Los números entre paréntesis corresponden a los valores mínimo y máximo

Números en negritas = albura

Números normales $=$ duramen 

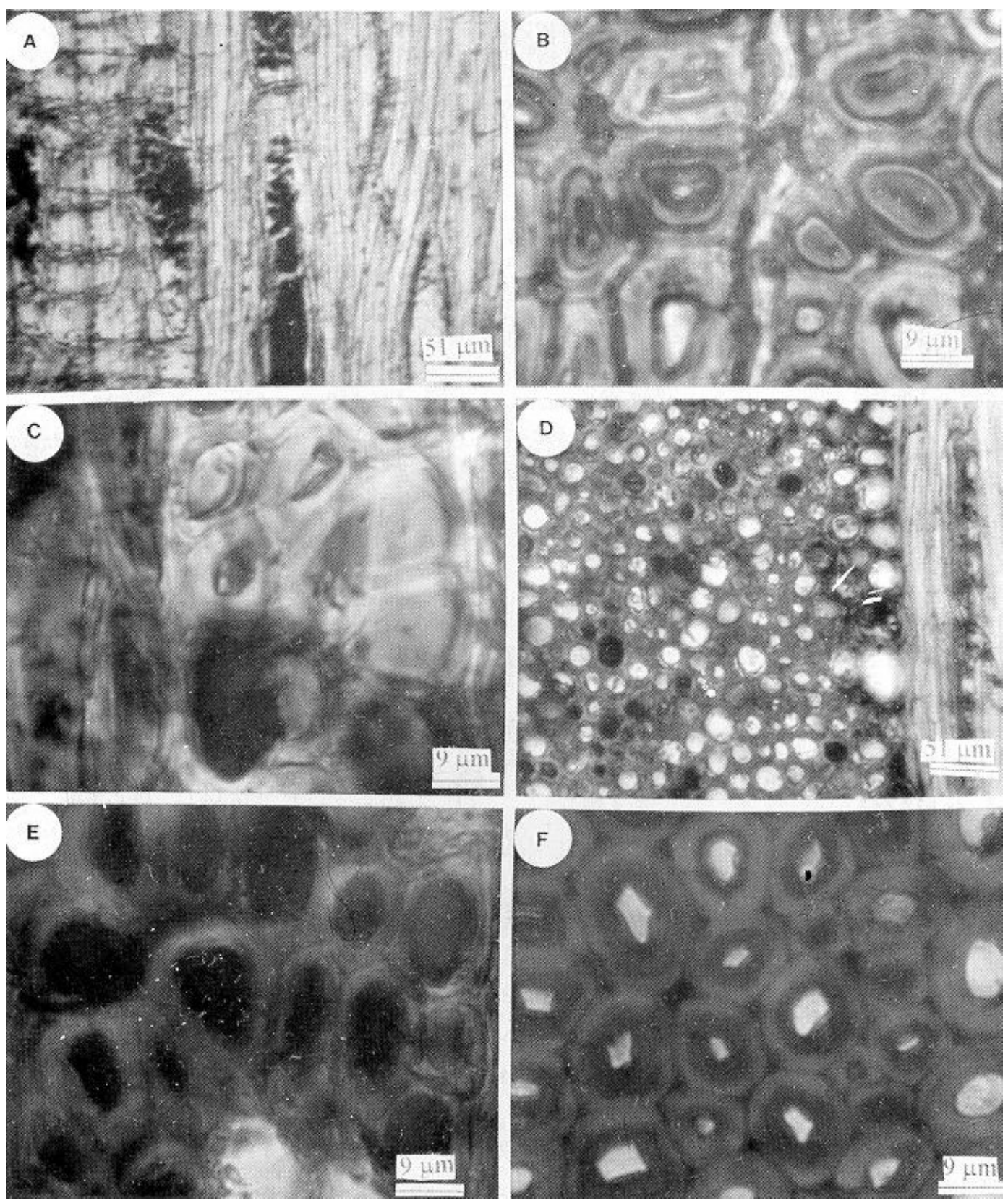

Lámina 1. Quercus glabrescens.

A) lugol en parénquima; B) fibras gelatinosas (ácido tánico-cloruro férrico); C) depósitos violáceos en parénquima (zinc-cloro-iodo); D) parénquima radial (azul de toluidina); E) fibras (fluroglucinol); F) fibras gelatinosas (azul de toluidina). 

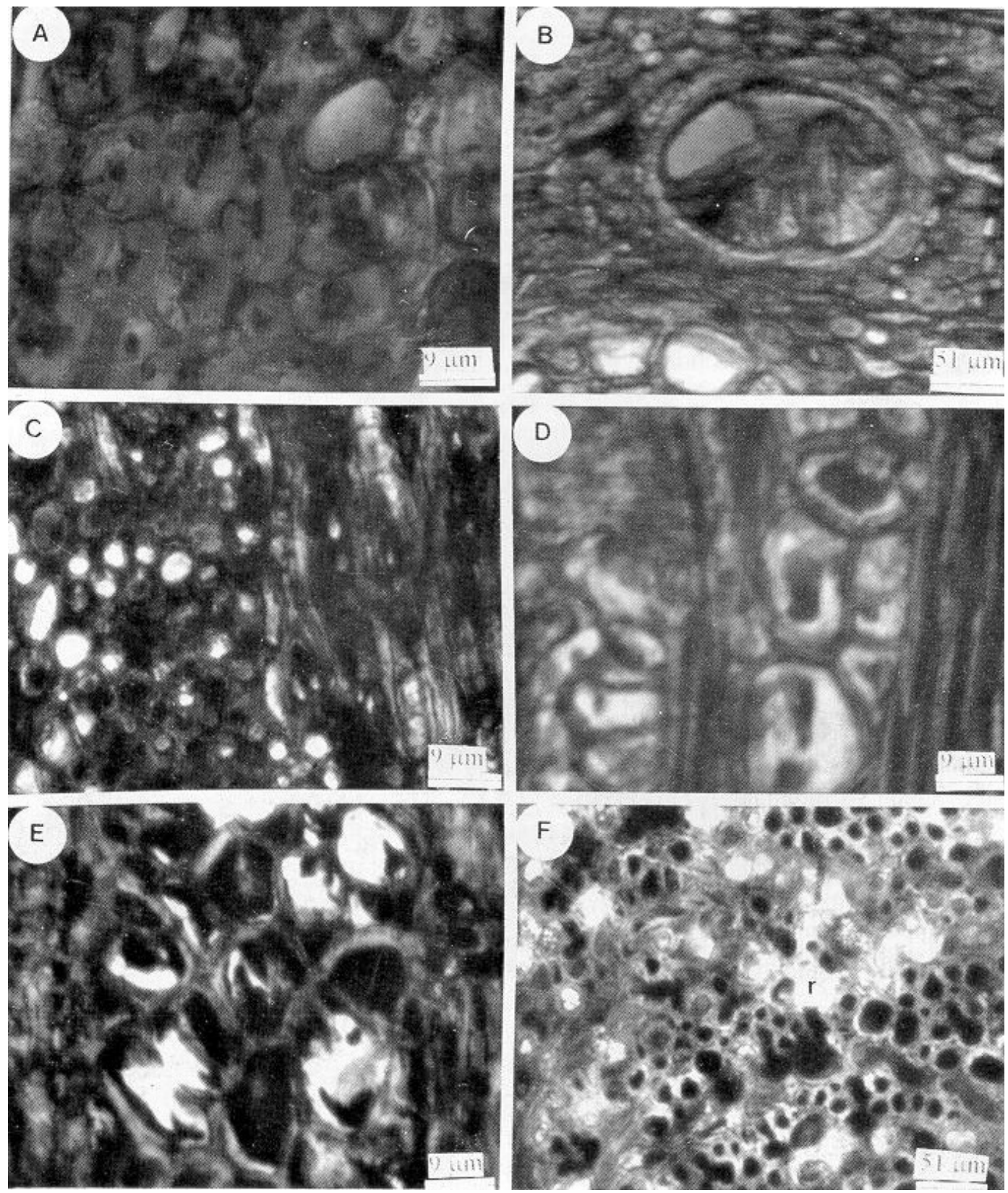

Lámina 2. Quercus potosina

A) fibras (zinc-cloro-iodo); b) lugol; C) y E) parénquima radial y axial (ácido tánico-cloruro férrico); D) fibras (fluroglucinol); F) parénquima radial (azul de toluidina). 

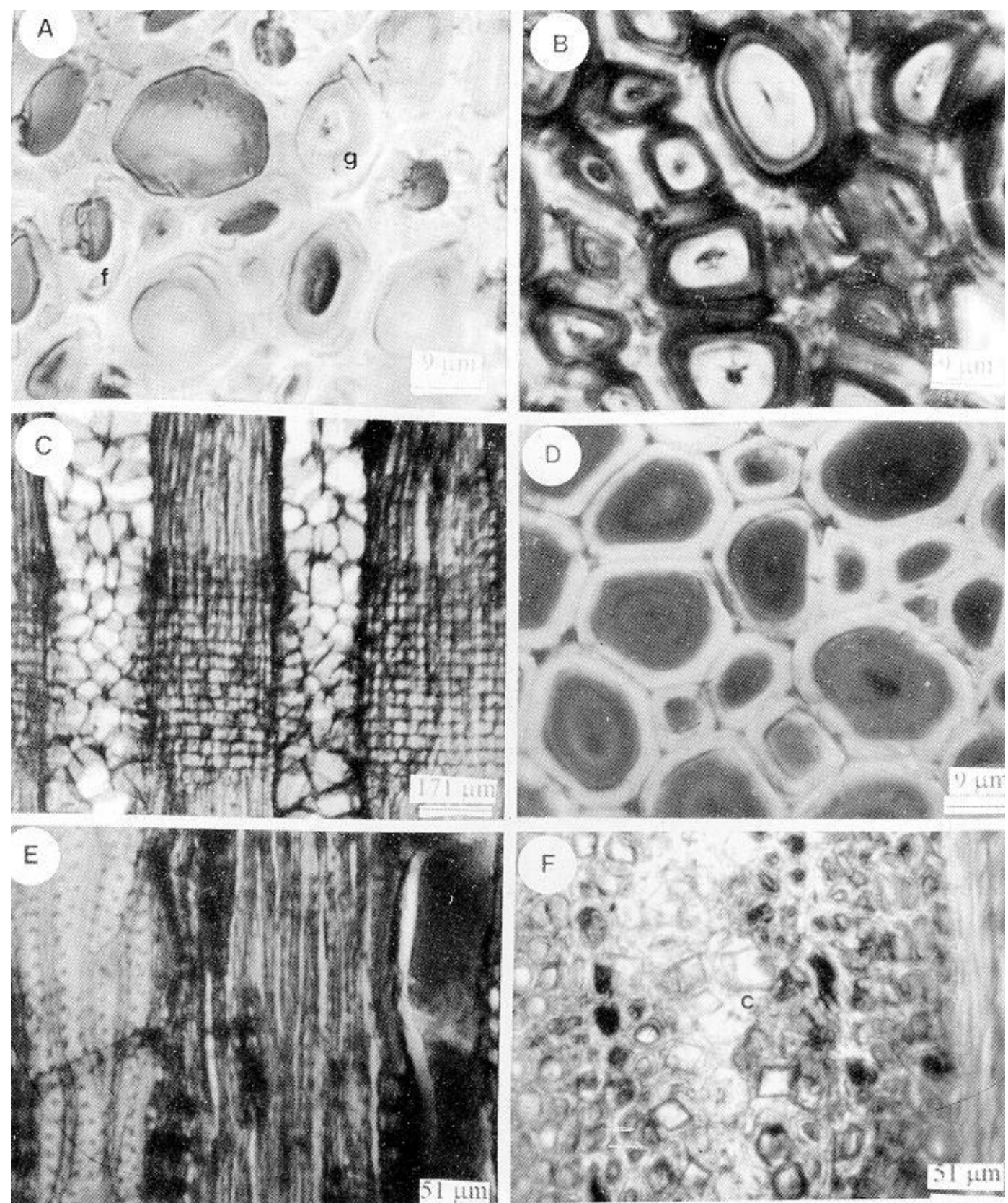

Lámina 3. Quercus eduardii

A) fibras (sin tinción); B) fibras gelatinosas (zinc-cloro-iodo); C) y D) tílides y fibras gelatinosas; E) elementos verticales (fluroglucinol); F) cristales romboidales en parénquima radial. 
Tabla 6b. Aspectos cuantitativos de parénquima axial y radial de Quercus glabrescens, Quercus potosina y Quercus eduardii.

\begin{tabular}{|c|c|c|c|c|c|c|}
\hline \multirow[b]{2}{*}{ ESPECIE } & \multicolumn{3}{|c|}{ PARENQUIMA AXIAL } & \multicolumn{3}{|c|}{ RADIOS } \\
\hline & $\begin{array}{c}\text { LONGITUD } \\
: \mathrm{m}\end{array}$ & $\begin{array}{c}\text { ANCHURA } \\
: \mathrm{m}\end{array}$ & $\begin{array}{c}\text { GROSOR } \\
\text { DE PARED } \\
: m\end{array}$ & $\begin{array}{c}\text { LONGITUD } \\
: \mathrm{m}\end{array}$ & $\begin{array}{c}\text { ANCHURA } \\
: \mathrm{m}\end{array}$ & $\begin{array}{c}\text { GROSOR } \\
\text { DE PARED } \\
: \mathrm{m}\end{array}$ \\
\hline \multirow{2}{*}{ Quercus glabrescens } & $\begin{array}{c}127 \\
(78-206)\end{array}$ & $\begin{array}{c}25 \\
(18-30)\end{array}$ & $\begin{array}{c}7 \\
(6-12)\end{array}$ & $\begin{array}{c}520 \\
(344-744)\end{array}$ & $\begin{array}{c}162 \\
(50-280)\end{array}$ & $\begin{array}{c}7 \\
(6-10)\end{array}$ \\
\hline & $\begin{array}{c}136 \\
(64-208)\end{array}$ & $\begin{array}{c}21 \\
(16-30)\end{array}$ & $\begin{array}{c}2 \\
(2-4)\end{array}$ & $\begin{array}{c}92 \\
(44-162)\end{array}$ & $\begin{array}{c}23 \\
(18-32)\end{array}$ & $\begin{array}{c}2 \\
(2-3)\end{array}$ \\
\hline \multirow{2}{*}{ Quercus potosina } & $\begin{array}{c}92 \\
(46-180)\end{array}$ & $\begin{array}{c}20 \\
(14-26)\end{array}$ & $\begin{array}{c}2 \\
(2-3)\end{array}$ & $\begin{array}{c}68 \\
(30-134)\end{array}$ & $\begin{array}{c}19 \\
(14-30)\end{array}$ & $\begin{array}{c}2 \\
(2-4)\end{array}$ \\
\hline & $\begin{array}{c}89 \\
(48-152)\end{array}$ & $\begin{array}{c}20 \\
(14-28)\end{array}$ & $\begin{array}{c}2 \\
(2-3)\end{array}$ & $\begin{array}{c}88 \\
(40-144)\end{array}$ & $\begin{array}{c}19 \\
(16-26)\end{array}$ & $\begin{array}{c}2 \\
(2-3)\end{array}$ \\
\hline \multirow{2}{*}{ Quercus eduardii } & $\begin{array}{c}126 \\
(62-232)\end{array}$ & $\begin{array}{c}27 \\
(18-40)\end{array}$ & $\begin{array}{c}2 \\
(2-4)\end{array}$ & $\begin{array}{c}80 \\
(24-162)\end{array}$ & $\begin{array}{c}22 \\
(16-32)\end{array}$ & $\begin{array}{c}3 \\
(2-4)\end{array}$ \\
\hline & $\begin{array}{c}128 \\
(64-204)\end{array}$ & $\begin{array}{c}26 \\
(18-41)\end{array}$ & $\begin{array}{c}3 \\
(2-4)\end{array}$ & $\begin{array}{c}69 \\
(24-142)\end{array}$ & $\begin{array}{c}20 \\
(16-34)\end{array}$ & $\begin{array}{c}3 \\
(2-4)\end{array}$ \\
\hline
\end{tabular}

Los números entre paréntesis corresponden a los valores mínimo y máximo Números en negritas = albura

Números normales $=$ duramen

Tabla 6c. Aspectos cuantitativos de traqueidas y fibrotraqueidas de Quercus glabrescens, Quercus potosina y Quercus eduardii.

\begin{tabular}{|c|c|c|c|c|c|c|}
\hline \multirow[b]{2}{*}{ ESPECIE } & \multicolumn{3}{|c|}{ TRAQUEIDAS } & \multicolumn{3}{|c|}{ 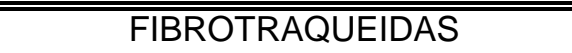 } \\
\hline & $\begin{array}{c}\text { LONGITUD } \\
: \mathrm{m}\end{array}$ & $\begin{array}{c}\text { DIAMETRO } \\
: \mathrm{m}\end{array}$ & $\begin{array}{c}\text { GROSOR } \\
\text { DE PARED } \\
: m\end{array}$ & $\begin{array}{c}\text { LONGITUD } \\
: \mathrm{m}\end{array}$ & $\begin{array}{c}\text { DIAMETRO } \\
: \mathrm{m}\end{array}$ & $\begin{array}{c}\text { GROSOR } \\
\text { DE PARED } \\
: \mathrm{m}\end{array}$ \\
\hline \multirow{2}{*}{ Quercus glabrescens } & $\begin{array}{c}857 \\
(600-1088)\end{array}$ & $\begin{array}{c}28 \\
(22-34\end{array}$ & $\begin{array}{c}6 \\
(4-8)\end{array}$ & $\begin{array}{c}979 \\
(704-1264)\end{array}$ & $\begin{array}{c}22 \\
(18-28)\end{array}$ & $\begin{array}{c}4 \\
(4-6)\end{array}$ \\
\hline & $\begin{array}{c}845 \\
(598-1078)\end{array}$ & $\begin{array}{c}27 \\
(22-33)\end{array}$ & $\begin{array}{c}6 \\
(4-8)\end{array}$ & $\begin{array}{c}980 \\
(710-1255)\end{array}$ & $\begin{array}{c}22 \\
(17-28)\end{array}$ & $\begin{array}{c}4 \\
(4-7)\end{array}$ \\
\hline \multirow{2}{*}{ Quercus potosina } & $\begin{array}{c}578 \\
(344-792)\end{array}$ & $\begin{array}{c}22 \\
(16-26)\end{array}$ & $\begin{array}{c}6 \\
(4-10)\end{array}$ & $\begin{array}{c}676 \\
(440-832)\end{array}$ & $\begin{array}{c}18 \\
(14-22)\end{array}$ & $\begin{array}{c}4 \\
(3-6)\end{array}$ \\
\hline & $\begin{array}{c}576 \\
(345-780)\end{array}$ & $\begin{array}{c}22 \\
(15-25)\end{array}$ & $\begin{array}{c}6 \\
(4-10)\end{array}$ & $\begin{array}{c}680 \\
(439-840)\end{array}$ & $\begin{array}{c}18 \\
(14-23)\end{array}$ & $\begin{array}{c}4 \\
(3-7)\end{array}$ \\
\hline \multirow{2}{*}{ Quercus eduardii } & $\begin{array}{c}682 \\
(432-968)\end{array}$ & $\begin{array}{c}31 \\
(24-44)\end{array}$ & $\begin{array}{c}7 \\
(4-10)\end{array}$ & $\begin{array}{c}821 \\
(464-1416)\end{array}$ & $\begin{array}{c}24 \\
(18-28)\end{array}$ & $\begin{array}{c}5 \\
(3-6)\end{array}$ \\
\hline & $\begin{array}{c}715 \\
(440-1008) \\
\end{array}$ & $\begin{array}{c}24 \\
(20-30) \\
\end{array}$ & $\begin{array}{c}5 \\
(4-10) \\
\end{array}$ & $\begin{array}{c}926 \\
(608-1248) \\
\end{array}$ & $\begin{array}{c}22 \\
(16-26) \\
\end{array}$ & $\begin{array}{c}4 \\
(3-6) \\
\end{array}$ \\
\hline
\end{tabular}

Los números entre paréntesis corresponden a los valores mínimo y máximo

Números en negritas = albura

Números normales $=$ duramen 


\section{DISCUSION}

El proceso de formación del duramen puede ser un aspecto muy variable a nivel de especie, sin embargo este proceso puede alterarse por incendios, disponibilidad de agua, humedad relativa y muy enfáticamente por las actividades de extracción casi exclusiva de pino en bosques de pino encino. Esta extracción por parte de los pobladores provoca que al tumbar, arrastrar y aclarar se modifique el proceso normal de crecimiento de los encinos que quedan en pie, lo que puede reflejarse de manera particular en cada caso. Estas actividades rompen con sistemas de microclima que son indispensables para mantener los tejidos meristemáticos secundarios en actividad normal. Este rompimiento genera que estos tejidos y las propias células verticales y horizontales que forman, se alteren y modifiquen la composición química de las paredes y los extractivos, las dimensiones celulares, la cantidad de tílides (en el caso de Quercus), e incluso la cantidad y calidad de fibras gelatinosas, cuyas capas G, pueden presentar composición química muy interesante. La madera de encino suele formar fibras gelatinosas como un tipo celular que no necesariamente indica presencia de madera de reacción (Tabla 2). Las especies de encino aquí estudiadas, presentaron no sólo fibras gelatinosas, sino modificaciones notables a estas fibras.

En este sentido, $Q$. glabrescens y $Q$. eduardiiprovienen de un bosque de neblina donde hay mayor cantidad de agua comparado con el sitio de donde proviene Q. potosina. El agua podría influir en la presencia de fibras gelatinosas (Láminas 1B y 3B) ya que es ésta la que permite estabilizar a los geles formados por pectinas y así tener cierta respuesta a la deformación. La función de estos geles está relacionada con la estabilización del agua y permite mantener el medio osmótico de la planta dando protección física para regular el transporte de metabolitos entre la planta y su medio (Vincent, 1982). Aunque la presencia y función de mayor o menor cantidad de fibras gelatinosas no ha sido estudiado, es claro que en una buena cantidad de las especies de Quercus existe una probable relación entre el agua (hábitat) y este tipo celular (Tabla 2).

Q. potosina proviene de un sitio en el que ha existido alta perturbación y por su topografía se ha generado un clima seco que está influyendo en un proceso mayor de lignificación de la pared celular que distingue a esta especie (Lámina 2D) de las otras dos. En este punto puede ser que dicha lignificación pareciera una estrategia contra la infección de microorganismos e insectos. Resalta que a pesar de ser un encino blanco, este individuo no presenta fibras gelatinosas que en general son características de los encinos y de individuos de la misma especie pero procedentes de otras localidades (Lámina 2A, Tabla 2).

Por otro lado, el bosque mesófilo del que provienen $Q$. glabrescens y $Q$. eduardii ha sufrido la tala inmoderada, que ha permitido que los árboles de esta región se infecten y presenten zonas que resaltan por los depósitos observados en la pared y en las cavidades con el fin de proveer defensa al individuo. Una respuesta a las infecciones está ejemplificada en $Q$. glabrescens, (Láminas 1B y E) en cuyo duramen la zona II presenta fibras gelatinosas que no se desarrollaron como las observadas en la albura y en cambio las paredes de este tipo de fibras, se lignificaron intensamente y en sus lúmenes también se depositaron claramente compuestos fenólicos de color rojo; también los demás elementos celulares se lignificaron y se presenta mayor cantidad de tílides en los vasos (Tabla 3), que en las otras dos zonas.

En general, debido a la presencia de hemicelulosas y sobre todo por estar las paredes celulares regular o densamente lignificadas, la celulosa presente en las paredes celulares de albura y duramen en los tres encinos, tuvo una baja reacción con la técnica de zinc-cloro-iodo (Jensen, 1962). 
Aunque en el duramen de las tres especies de encinos se esperaba encontrar componentes distintos a los polisacáridos presentes en la albura, se observaron carbohidratos violáceos y de color pardorosados en las fibras, vasos, parénquima axial y radial de todo el xilema secundario. De acuerdo con Jensen (1962), en el método de lugol, la longitud de la molécula de almidón aparentemente determina el color final de la reacción; para las moléculas más cortas, el color es más rojo y para las moléculas más largas, el color es más azul. Debido al color de los carbohidratos observados en las tres especies de encinos y a que no tienen consistencia granulosa, podrían ser carbohidratos no estructurados como ocurre en el grano de almidón.

En el duramen de $Q$. glabrescens las paredes de la zona I y de la III presentaron una composición química muy parecida (tabla 3); estas dos zonas están separadas por la zona II que está más lignificada. En la zona I, a diferencia de la zona II, el duramen presenta pocas células de parénquima axial y radial con carbohidratos violáceos. Por otro lado, la presencia de pectinas esterificadas es mayor en las paredes celulares de la zona II que en las zonas I y III.

Las fibras gelatinosas observadas en $Q$. glabrescens y $Q$. eduardii tienen una pared gelatinosa cuyas capas se distinguen por la cantidad de pectatos de calcio y de hemicelulosas que las conforman.

En la albura de las tres especies, se observaron pequeñas gotas de lípidos; $Q$. glabrescens las presenta en el parénquima radial, $Q$. eduardii en el parénquima axial y radial y $Q$. potosina las presenta en el parénquima axial, parénquima radial y además en pocas tílides.

En la albura de $Q$. potosina algunas células del parénquima radial contienen oligómeros de catequinas y leucoantocinidinas (depósitos pardo - rojizos) en el lumen y en algunas tílides del duramen. En la albura de Q. eduardii estos oligómeros se observaron en pocas tílides y en algunas células del parénquima axial y radial mientras que también en el duramen pocas fibras y vasos los contienen. En contraste con estas dos especies, $Q$. glabrescens no presenta este tipo de contenidos (tablas 3, 4 y 5).

Las tres especies de encino presentan algunas semejanzas en sus características como son: presencia de tílides, dimensiones similares de diámetro de fibras, grosor de paredes de fibras, vasos, paréquima axial y radial así como presencia de traqueidas y fibrotraqueidas. Quercus potosina resalta de los otras dos especies por presentar las menores dimensiones en: longitud de fibras, vasos, traqueidas y fibrotraqueidas, así como en diámetro de vasos y longitud de células de parénquima (tablas 6a-c).

Q. potosina resalta por tener en la albura células especializadas del parénquima axial que contienen cristales romboidales. Aunque $Q$. eduardii también presenta cristales como $Q$. potosina, éstos son pocos y se localizan en los rayos multiseriados que contienen procesos pétreos.

Se observó que en general las dimensiones sobre todo de las fibras y los vasos de la albura tienden a ser mayores y disminuyen hacia el duramen, principalmente en $Q$. eduardii y $Q$. glabrescens. Sin embargo esto no ocurre para el parénquima axial y radial y las traqueidas y fibrotraqueidas. La disminución del tamaño de los vasos en el duramen, puede explicarse por la influencia de la fuerza centrípeta ejercida por el crecimiento secundario de los árboles, pero no explica los valores de las dimensiones para fibrotraqueidas, traqueidas y parénquima en albura y duramen.

La calidad de las paredes celulares depende del tipo y cantidad de componentes químicos que presenten. En el caso de $Q$. 
glabrescens, el hecho de presentar paredes celulares más lignificadas en ciertas regiones del tejido y fibras gelatinosas densamente compuestas por sustancias pécticas podría influir en una menor o mayor resistencia a las fuerzas mecánicas. Por ejemplo, Q. potosina, por presentar zonas de tejido densamente lignificado, ausencia de elementos estabilizadores (fibras gelatinosas) y elementos más cortos puede presentar valores elásticos menores que el resto de las especies. Esto significa que las células pueden transformar polisacáridos en depósitos de compuestos fenólicos y taninos en cantidades sorprendentes (Lámina 2 E y F).

La lignificación puede disminuir la porosidad y la permeabilidad al agua en las paredes, no sólo de la madera, sino también de la corteza, lo que las hace más rígidas, pero factores desestabilizadores externos como por ejemplo los procesos de desertificación y la consecuente disminución de agua accesible, puede provocar fracturas a nivel de peridermis y por tanto, ataque de insectos que promueven secuencias infecciosas posteriores, hacia el interior del tallo secundario.

En general, se observa que el cambio de albura a duramen de las tres especies es muy similar en cuanto a que presentan un duramen poco marcado, por lo que la transformación de polisacaridos y lípidos es gradual ya es posible encontrar en algunas células de parénquima axial de duramen, contenidos violáceos. El mecanismo de formación de duramen ("duraminización") en estas especies resulta muy parecido al de otras especies de encinos en las que el duramen no está perfectamente marcado como en muchas especies tropicales. Sus estrategias de duraminización están orientadas mayormente a la formación de tílides, depósitos de polisacáridos y compuestos fenólicos y en menor escala al engrosamiento y alargamiento de las paredes celulares.

\section{CONCLUSIONES}

La calidad de las paredes celulares del xilema secundario en las tres especies estudiadas está afectada por el tipo y la cantidad de componentes químicos, por sus dimensiones celulares y por las estrategias de defensa que han desarrollado estos individuos, para sobrevivir en ambientes tan alterados como son las zonas en que fueron cortados los árboles estudiados. El concepto de calidad de pared celular estará relacionado con el uso al que se destine la madera de cierta especie bajo condiciones estrictas de silvicultura, donde la cantidad de agua, el suelo y el ambiente están "controlados". En ambientes en los cuales lo que resalta es la alteración, será difícil discutir esto y, sí las estrategias seguidas por los individuos para sobrevivir responden realmente a los mismos fenómenos que afectan la formación de la madera. Los fenómenos macroscópicos son el producto de un comportamiento microscópico y aunque los valores mecánicos pueden resumir la calidad, también estarán afectados por el ambiente en que crecen.

\section{RECONOCIMIENTOS}

El presente trabajo fue financiado por la Universidad Autónoma MetropolitanaIztapalapa. Se agradece al Ejido de Huayacocotla y en especial al Ing. Raúl García de la Cadena por su colaboración. Asimismo, al Dr. Raymundo Dávalos Sotelo, del Instituto de Ecología A.C. por las facilidades brindadas para el procesamiento del material. Al mismo tiempo se agradece a los revisores anónimos por sus puntuales observaciones que, sentimos, sirvieron para reconsiderar algunos de los aspectos de la histoquímica de la madera.

\section{REFERENCIAS}

Baqui, S.; J. J. Shah; R. C. Pandalai y I. L. Kothari.1979. Histochemical changes during transition from sapwood to 
heartwood in Melia azedarach young branch. Indian Journal of Experimental Biology 17:1032-1037.

Datta, S.K. y A. Kumar. 1987. Histochemical studies of the transition from sapwood to heartwood in Tectona grandis. IAWA Bull. n. s. 8: 363-368.

De la Paz Pérez O., C. 1982. Anatomía de la madera de cinco especies de encinos de Durango. Bol. Téc. Inst. Nac. Inv. For. No. 43. México. 35 p.

Donaldson, L.A. 1992. Lignin distribution during latewood formation in Pinus radiata D. Don. IAWA Bull. n. s. 13: 381-387.

Jensen,W.A.1962. Botanical histochemistry. W. H. Freeman, San Francisco. EUA.
Johansen, D.A. 1940. Plant microtechnique. McGraw Hill Book Co., New York. EUA.

Krishnamurthy, K. V. 1988. Methods in plant histochemistry. Bharathidasan University. S. Viswanathan Press. India.

Quintanar, A., L. Rivera, A. Torre-Blanco y S. Rebollar D. 1997. Comparative histochemistry and cell morphology of sapwood and heartwood of Gliricidia sepium (Fabaceae). Rev. Biol. Trop. 45:1005-1011.

Shah, J.J., S. Baqui, R.C. Pandalai y K.P. Patel. 1981. Histochemical changes in Acacia nilotica L. during transition from sapwood to heartwood. IAWA Bull. n. s. 2:31-36.

Vincent, J. 1982. Structural biomaterials. Halsted Press. New York. EUA 